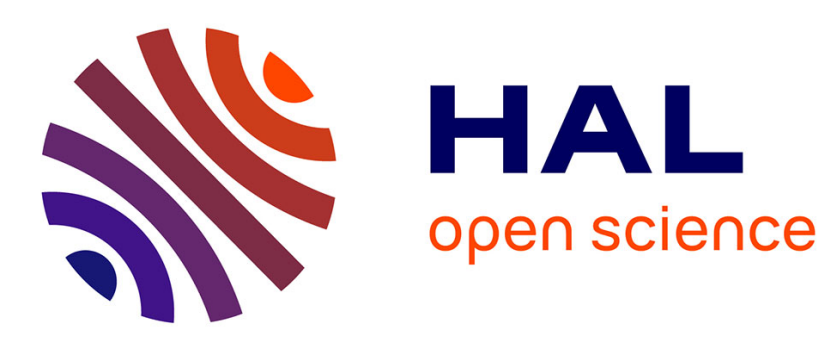

\title{
On the geometry of predictive control with nonlinear constraints
}

\author{
Sorin Olaru, Didier Dumur, Simona Dobre
}

\section{To cite this version:}

Sorin Olaru, Didier Dumur, Simona Dobre. On the geometry of predictive control with nonlinear constraints. Joaquim Filipe, Juan Andrade Cetto and Jean-Louis Ferrier. Informatics in Control, Automation and Robotics. Selected Papers from the International Conference on Informatics in Control, Automation and Robotics 2007, Springer, pp.301-314, 2008, Lecture Notes in Electrical Engineering, 10.1007/978-3-540-85640-5_23. hal-00322997

\section{HAL Id: hal-00322997 https://hal.science/hal-00322997}

Submitted on 19 Sep 2009

HAL is a multi-disciplinary open access archive for the deposit and dissemination of scientific research documents, whether they are published or not. The documents may come from teaching and research institutions in France or abroad, or from public or private research centers.
L'archive ouverte pluridisciplinaire HAL, est destinée au dépôt et à la diffusion de documents scientifiques de niveau recherche, publiés ou non, émanant des établissements d'enseignement et de recherche français ou étrangers, des laboratoires publics ou privés. 


\title{
On the geometry of predictive control with nonlinear constraints
}

\author{
Sorin Olaru ${ }^{1}$, Didier Dumur ${ }^{1}$, and Simona Dobre ${ }^{2}$ \\ 1 Automatic Control Department, SUPELEC, 3 rue Joliot Curie, Gif-sur-Yvette, \\ France \\ \{sorin.olaru;didier.dumur\}@supelec.fr, \\ 2 CRAN, BP 239, 54506 Vandœuvre-lès-Nancy, France \\ simona.dobre@cran.uhp-nancy.fr
}

\begin{abstract}
This paper proposes a geometrical analysis of the polyhedral feasible domains for the predictive control laws under constraints. The state vector is interpreted as a vector of parameters for the optimization problem to be solved at each sampling instant and its influence can be fully described by the use of parameterized polyhedra and their dual constraints/generators representation. The construction of the associated explicit control laws at least for linear or quadratic cost functions can thus receive fully geometrical solutions. Convex nonlinear constraints can be approximated using a description based on the parameterized vertices. In the case of nonconvex regions the explicit solutions can be obtained using Voronoi partitions based on a collection of points distributed over the borders of the feasible domain.
\end{abstract}

\section{Introduction}

The philosophy behind Model-based Predictive Control (MPC) is to exploit in a "receding horizon" manner the simplicity of the open-loop optimal control. The control action $u_{t}$ for a given state $x_{t}$ is obtained from the control sequence $\mathbf{k}_{u}^{*}=\left[u_{t}^{T}, \ldots, u_{t+N-1}^{T}\right]^{T}$ as a result of the optimization problem:

$$
\begin{array}{ll}
\min _{\mathbf{k}_{u}} & \varphi\left(x_{t+N}\right)+\sum_{k=0}^{N-1} l\left(x_{t+k}, u_{t+k}\right) \\
\text { subj. to }: & x_{t+1}=f\left(x_{t}\right)+g\left(x_{t}\right) u_{t} ; \\
& h\left(x_{t}, \mathbf{k}_{u}\right) \leq 0
\end{array}
$$

constructed for a finite prediction horizon $N$, cost per stage $l($.$) , terminal weight$ $\varphi($.$) , the system dynamics described by f(),. g($.$) and the constraints written in$ a compact form using elementwise inequalities on functions linking the states and the control actions, $h($.$) .$

The control sequence $\mathbf{k}_{u}^{*}$ is optimal for an initial condition $-x_{t}$ and produces an open-loop trajectory which contrasts with the need for a feedback control law. This drawback is overcome by solving the local optimization (1) for every encountered (measured) state, thus indirectly producing a state feedback law. 
For the optimization problem (1) within MPC, the current state serves as an initial condition and influences both the objective function and the topology of the feasible domain. Globally, the system state can be interpreted as a vector of parameters, and the problems to be solved are part of the multiparametric optimization programming family. From the cost function point of view, the parametrization is somehow easier to deal with and eventually can be entirely translated toward the set of constraints to be satisfied (the MPC literature contains references to schemes based on suboptimality or even to algorithms restraining the demands to feasible solution of the receding horizon optimization [1]). Unfortunately, similar observation cannot be made about the feasible domain and its adjustment with respect to the parameters evolution. The optimal solution is thus often influenced by the constraints activation, the process being forced to operate at the designed constraints for best performance. The distortion of the feasible domain during the parameters evolution will consequently affect the structure of the optimal solution. Starting from this observation the present paper focuses on the analysis of the geometry of the domains described by the MPC constraints.

The structure of the feasible domain is depending on the model and the set of constraints taken into consideration in (1). If the model is linear, the linear constraints on inputs and states can be easily expressed by a system of linear inequalities. In the case of nonlinear systems, these properties are lost but there are several approaches to transform the dynamics to those of a linear system over the operating range as for example by piecewise linear approximation, feedback linearisation or the use of time-varying linear models.

In the present paper, the feasible domains will be analyzed with a focus on the parametrization mainly upon the concept of parameterized polyhedra [2], which appears in the MPC formulations like:

$$
\begin{gathered}
\min _{\mathbf{k}_{u}} F\left(x_{t}, \mathbf{k}_{u}\right) \\
\text { subj. to : }\left\{\begin{array}{c}
A_{i n} \mathbf{k}_{u} \leq b_{i n}+B_{i n} x_{t} \\
A_{e q} \mathbf{k}_{u}=b_{e q}+B_{e q} x_{t} \\
h\left(x_{t}, \mathbf{k}_{u}\right) \leq 0
\end{array}\right.
\end{gathered}
$$

where the objective function $F\left(x_{t}, \mathbf{k}_{u}\right)$ is usually linear or quadratic. Secondly it will be shown that the optimization problem may take advantage during the real-time implementation from the construction of the explicit solution.

In the presence of nonlinearities $h\left(x_{t}, \mathbf{k}_{u}\right) \leq 0$ two cases can be treated:

- feasible domain are convex - the approximation in terms of parameterized polyhedra leads to an approximate explicit solution using the same arguments as for the exact solutions;

- feasible domain is non-convex - an algorithmic construction of explicit control laws upon Voronoi partition of the parameters space will be used.

In the following, Section 2 introduces the basic concepts related to the parameterized polyhedra. Section 3 presents the use of the feasible domain analysis for the construction of the explicit solution for linear and quadratic objective functions. In Section 4 an extension to nonlinear type of constraints is addressed. 


\section{Parametrization of polyhedral domains}

\subsection{Double representation}

A mixed system of linear equalities and inequalities defines a polyhedron [3]. In the parameter free case, it is represented by the equivalent dual (Minkowski) formulation:

$$
\begin{aligned}
& \mathcal{P}=\left\{\mathbf{k}_{u} \in \mathbb{R}^{p} \mid A_{e q} \mathbf{k}_{u}=b_{e q} ; A_{\text {in }} \mathbf{k}_{u} \leq b_{\text {in }}\right\} \\
& \Longleftrightarrow \mathcal{P}=\underbrace{\text { conv.hull } \mathbf{V}+\text { cone } \mathbf{R}+\text { lin.space } \mathbf{L}}_{\text {generators }}
\end{aligned}
$$

where conv.hull $\mathbf{V}$ denotes the set of convex combinations of vertices $\mathbf{V}=\left\{\mathbf{v}_{1}, \ldots, \mathbf{v}_{\vartheta}\right\}$, cone $\mathbf{R}$ denotes nonnegative combinations of unidirectional rays in $\mathbf{R}=\left\{\mathbf{r}_{1}, \ldots, \mathbf{r}_{\rho}\right\}$ and lin.space $\mathbf{L}=\left\{\mathbf{l}_{1}, \ldots, \mathbf{l}_{\lambda}\right\}$ represents a linear combination of bidirectional rays (with $\vartheta, \rho$ and $\lambda$ the cardinals of the related sets). This dual representation [4] in terms of generators can be rewritten as:

$$
\begin{gathered}
\mathcal{P}=\left\{\mathbf{k}_{u} \in \mathbb{R}^{p} \mid \mathbf{k}_{u}=\sum_{i=1}^{\vartheta} \alpha_{i} \mathbf{v}_{i}+\sum_{i=1}^{\rho} \beta_{i} \mathbf{r}_{i}+\sum_{i=1}^{\lambda} \gamma_{i} \mathbf{l}_{i} ;\right. \\
\left.0 \leq \alpha_{i} \leq 1, \sum_{i=1}^{\vartheta} \alpha_{i}=1, \beta_{i} \geq 0, \forall \gamma_{i}\right\}
\end{gathered}
$$

with $\alpha_{i}, \beta_{i}, \gamma_{i}$ the coefficients describing the convex, non-negative and linear combinations in (3). Numerical methods like the Chernikova algorithm [5] are implemented for constructing the double description, either starting from constraints (3) either from the generators (4) representation.

\subsection{The parametrization}

A parameterized polyhedron [6] is defined in the implicit form by a finite number of inequalities and equalities with the note that the affine part depends linearly on a vector of parameters $x \in \mathbb{R}^{n}$ for both equalities and inequalities:

$$
\begin{aligned}
\mathcal{P}(x) & =\left\{\mathbf{k}_{u}(x) \in \mathbb{R}^{p} \mid A_{e q} \mathbf{k}_{u}=B_{e q} x+b_{e q} ; A_{i n} \mathbf{k}_{u} \leq B_{i n} x+b_{i n}\right\} \\
& =\left\{\mathbf{k}_{u}(x) \mid \mathbf{k}_{u}(x)=\sum_{i=1}^{\vartheta} \alpha_{i}(x) \mathbf{v}_{i}(x)+\sum_{i=1}^{\rho} \beta_{i} \mathbf{r}_{i}+\sum_{i=1}^{\lambda} \gamma_{i} \mathbf{l}_{i}\right\} \\
& \leq \alpha_{i}(x) \leq 1, \sum_{i=1}^{\vartheta} \alpha_{i}(x)=1, \beta_{i} \geq 0, \forall \gamma_{i} .
\end{aligned}
$$

This dual representation of the parameterized polyhedral domain reveals the fact that only the vertices are concerned by the parametrization (resulting the so-called parameterized vertices $-\mathbf{v}_{i}(x)$ ), whereas the rays and the lines do not change with the parameters' variation. In order to effectively use the generators representation in (5), several aspects have to be clarified regarding the parametrization of the vertices (see for exemple [6]). The idea is to identify the parameterized polyhedron by a non-parameterized one in an augmented space:

$$
\tilde{\mathcal{P}}=\left\{\left[\begin{array}{c}
\mathbf{k}_{u} \\
x
\end{array}\right] \in \mathbb{R}^{p+n} \mid\left[A_{e q} \mid-B_{e q}\right]\left[\begin{array}{c}
\mathbf{k}_{u} \\
x
\end{array}\right]=b_{e q} ;\left[A_{i n} \mid-B_{i n}\right]\left[\begin{array}{c}
\mathbf{k}_{u} \\
x
\end{array}\right] \leq b_{i n}\right\}
$$


The original polyhedron in (5) can be found for any particular value of the parameters vector $x$ through $P(x)=\operatorname{Proj}_{\mathbf{k}_{u}}(\tilde{P} \cap H(x))$, for any given hyperplane $H\left(x_{0}\right)=\left\{\left(\begin{array}{c}\mathbf{k}_{u} \\ x\end{array}\right) \in \mathbb{R}^{p+n} \mid x=x_{0}\right\}$ and using $\operatorname{Proj}_{\mathbf{k}_{u}}($.$) as the projection from$ $\mathbb{R}^{p+n}$ to the first $p$ coordinates $\mathbb{R}^{p}$.

Within the polyhedral domains $\tilde{\mathcal{P}}$, the correspondent of the parameterized vertices in (5) can be found among the faces of dimension $n$. After enumerating these $n$-faces: $\left\{F_{1}^{n}(\tilde{\mathcal{P}}), \ldots F_{j}^{n}(\tilde{\mathcal{P}}), \ldots, F_{\varsigma}^{n}(\tilde{\mathcal{P}})\right\}$, one can write: $\forall i, \exists j \in$ $\{1, \ldots, \varsigma\}$ s.t. $\left[\mathbf{v}_{i}(x)^{T} x^{T}\right]^{T} \in F_{j}^{n}(\tilde{\mathcal{P}})$ or equivalently:

$$
\mathbf{v}_{i}(x)=\operatorname{Proj}_{\mathbf{k}_{u}}\left(F_{j}^{n}(\tilde{\mathcal{P}}) \cap H(x)\right)
$$

From this relation it can be seen that not all the $n$-faces correspond to parameterized vertices. However it is still easy to identify those which can be ignored in the process of construction of parameterized vertices based on the relation $\operatorname{Proj}_{x}\left(F_{j}^{n}(\tilde{P})\right)<n$ with $\operatorname{Proj}_{x}($.$) the projection from \mathbb{R}^{p+n}$ to the last $n$ coordinates $\mathbb{R}^{n}$ (corresponding to the parameters' space). Indeed the projections are to be computed for all the $n$-faces, those which are degenerated are to be discarded and all the others are stored as validity domains - $D_{\mathbf{v}_{i}} \in \mathbb{R}^{n}$, for the parameterized vertices that they are identifying:

$$
D_{\mathbf{v}_{i}}=\operatorname{Proj}_{n}\left(F_{j}^{n}(\tilde{P})\right)
$$

Once the parameterized vertices identified and their validity domain stored, the dependence on the parameters vector can be found using the supporting hyperplanes for each $n$-face:

$$
\mathbf{v}_{i}(x)=\left[\begin{array}{c}
A_{e q} \\
\bar{A}_{i n_{j}}
\end{array}\right]^{-1}\left[\begin{array}{c}
B_{e q} \\
\bar{B}_{i n_{j}}
\end{array}\right] x+\left[\begin{array}{c}
b_{e q} \\
\bar{b}_{i n_{j}}
\end{array}\right]
$$

where $\bar{A}_{i n_{j}}, \bar{B}_{i n_{j}}, \bar{b}_{i n_{j}}$ represent the subset of the inequalities, satisfied by saturation for $F_{j}^{n}(\tilde{P})$. The inversion is well defined as long as the faces with degenerate projections are discarded.

\subsection{The interpretation from the predictive control point of view}

The double representation of the parameterized polyhedra offers a complete description of the feasible domain for the MPC law as long as this is based on a multiparametric optimization with linear constraints. Using the generators representation one can compute the region of the parameters space where no parameterized vertex is defined:

$$
\aleph=\mathbb{R}^{n} \backslash\left\{\cup D_{\mathbf{v}_{i}} ; i=1 \ldots \vartheta\right\}
$$


representing the set of infeasible states for which no control sequence can be designed due to the fact that the limitations are overly constraining. As a consequence the complete description of the infeasibility is obtained.

The vertices of the feasible domain cannot be expressed as convex combinations of other distinct points and, due to the fact that from the MPC point of view, they represent sequences of control actions, one can interpret them in terms of extremal performances of the controlled system (for example in the tracking applications the maximal/minimal admissible setpoint [7]).

\section{Toward explicit solutions for polyhedral domains}

In the case of sufficiently large memory resources, construction of the explicit solution for the multiparametric optimization problem (2) can be an interesting alternative to the iterative optimization routines. In this direction recent results were presented at least for the case of linear and quadratic cost functions (see $[8],[9],[10],[11],[12])$. In the following it will be shown that a geometrical approach based on the parameterized polyhedra can bring a useful insight as well.

\subsection{Linear cost function}

The linear cost functions are extensively used in connection with model based predictive control and especially for robust case ([13], [14]). In a compact form, the multiparametric optimization problem is:

$$
\begin{aligned}
& \mathbf{k}_{u}{ }^{*}\left(x_{t}\right)=\min _{\mathbf{k}_{u}} f^{T} \mathbf{k}_{u} \\
& \text { subject to } A_{i n} \mathbf{k}_{u} \leq B_{i n} x_{t}+b_{i n}
\end{aligned}
$$

The problem deals with a polyhedral feasible domain which can be described as previously in a double representation. Further the explicit solution can be constructed based on the relation between the parameterized vertices and the linear cost function (as in [5]). The next result resumes this idea.

Proposition: The solution for a multiparametric linear problem is characterized as follows:

a) For the subdomain $\aleph \in \mathbb{R}^{n}$ where the associated parameterized polyhedron has no valid parameterized vertex the problem is infeasible;

b) If there exists a bidirectional ray $\mathbf{l}$ such that $f^{T} \mathbf{l} \neq 0$ or a unidirectional ray $\mathbf{r}$ such that $f^{T} \mathbf{r} \leq 0$, then the minimum is unbounded;

c) If all bidirectional rays $\mathbf{l}$ are such that $f^{T} \mathbf{l}=0$ and all unidirectional rays $\mathbf{r}$ are such that $f^{T} \mathbf{r} \geq 0$ then there exists a cutting of the parameters in zones where the parameterized polyhedron has a regular shape $\bigcup_{j=1 \ldots \rho} R_{j}=\mathbb{R}^{n}-\aleph$. For each region $R_{j}$ the minimum is computed with respect to the given linear cost function and for all the valid parameterized vertices:

$$
\underline{m}(x)=\min \left\{f^{T} \mathbf{v}_{i}(x) \mid \mathbf{v}_{i}(x) \text { vertex of } \mathcal{P}(x)\right\}
$$


The minimum $\underline{m}(x)$ is attained by constant subsets of parameterized vertices of $\mathcal{P}(x)$ over a finite number of polyhedral zones in the parameters space $R_{i j}$ $\left(\cup R_{i j}=R_{j}\right)$. The complete optimal solution of the multiparametric optimization is given for each $R_{i j}$ by:

$$
S_{R_{i j}}(x)=\text { conv.hull }\left\{\mathbf{v}_{1}^{*}(x), \ldots, \mathbf{v}_{s}^{*}(x)\right\}+\text { cone }\left\{\mathbf{r}_{1}^{*}, \ldots, \mathbf{r}_{r}^{*}\right\}+\text { lin.space } \mathcal{P}
$$

where $\mathbf{v}_{i}^{*}$ are the vertices corresponding to the minimum $\underline{m}(x)$ over $R_{i j}$ and $\mathbf{r}_{i}^{*}$ are such that $f^{T} \mathbf{r}_{i}^{*}=0_{\square}$

This result provides the entire family of solutions for the linear multiparametric optimization, even for the cases where this family is not finite (for example there are several vertices attaining the minimum). For the control point of view a continuous piecewise candidate is preferred, eventually by minimizing the number of partitions in the parameters space [15].

\subsection{Quadratic cost function}

The case of a quadratic cost function is one of the most popular for the linear nominal MPC. The explicit solution based on the exploration of the parameters space $([9],[11],[12])$ is extensively studied lately. Alternative methods based on geometrical arguments or dynamic programming ([10], [8]) improved also the awareness of the explicit MPC formulations. The parameterized polyhedra can serve as a base in the construction of such explicit solution [2], for a quadratic multiparametric problem:

$$
\begin{aligned}
& \mathbf{k}_{u}{ }^{*}\left(x_{t}\right)=\arg \min _{\mathbf{k}_{u}} \mathbf{k}_{u}^{T} H \mathbf{k}_{u}+2 \mathbf{k}_{u}{ }^{T} F x_{t} \\
& \text { subject to } A_{i n} \mathbf{k}_{u} \leq B_{i n} x_{t}+b_{i n}
\end{aligned}
$$

In this case the main idea is to consider the unconstrained optimum:

$$
\mathbf{k}_{u}^{s c}\left(x_{t}\right)=H^{-1} F x_{t}
$$

and its position with respect to the feasible domain given by a parameterized polyhedron as in (5).

If a simple transformation is performed:

$$
\tilde{\mathbf{k}}_{u}=H^{1 / 2} \mathbf{k}_{u}
$$

then the isocost curves of the quadratic function are transformed from ellipsoid into circles centered in $\tilde{\mathbf{k}}_{u}^{s c}\left(x_{t}\right)=H^{-1 / 2} F x_{t}$. Further one can use the Euclidean projection in order to retrieve the multiparametric quadratic explicit solution.

Indeed if the unconstrained optimum $\tilde{\mathbf{k}}_{u}^{s c}\left(x_{t}\right)$ is contained in the feasible domain $\tilde{\mathcal{P}}\left(x_{t}\right)$ then it is also the solution of the constrained case, otherwise existence and uniqueness are assured as follows:

Proposition: For any exterior point $\tilde{\mathbf{k}}_{u}\left(x_{t}\right) \notin \tilde{\mathcal{P}}\left(x_{t}\right)$, there exists an unique point characterized by a minimal distance with respect to $\tilde{\mathbf{k}}_{u}^{s c}\left(x_{t}\right)$. This point satisfies:

$$
\left(\tilde{\mathbf{k}}_{u}^{s c}\left(x_{t}\right)-\tilde{\mathbf{k}}_{u}^{*}\left(x_{t}\right)\right)^{T}\left(\tilde{\mathbf{k}}_{u}-\tilde{\mathbf{k}}_{u}^{*}\left(x_{t}\right)\right) \leqslant 0, \forall \tilde{\mathbf{k}}_{u} \in \tilde{\mathcal{P}}\left(x_{t}\right) \square
$$


The construction mechanism uses the parameterized vertices in order to split the regions neighboring the feasible domain in zones characterized by the same type of projection.

\section{Generalization to nonlinear constraints}

If the feasible domain is described by a mixed linear/nonlinear set of constraints then the convexity properties are lost and a procedure for the construction of exact explicit solutions does not exist for the general case.

Consider now the case of mixed type of constraints (linear/nonlinear):

$$
\begin{aligned}
\mathbf{k}_{u}^{*}=\underset{\mathbf{k}_{u}}{\arg \min } 0.5 \mathbf{k}_{u}^{T} H \mathbf{k}_{u}+\mathbf{k}_{u}^{T} F x \\
\\
\left\{\begin{array}{c}
h\left(x, \mathbf{k}_{u}\right) \leqslant 0 \\
A_{\text {in }} \mathbf{k}_{u} \leq b_{\text {in }}+B_{\text {in }} x
\end{array}\right.
\end{aligned}
$$

\subsection{Optimality conditions for nonlinear constraints}

Let $\bar{x}$ be a feasible parameter vector. The KKT optimality conditions can still be formulated as:

- Primal feasibility:

$$
\left\{\begin{array}{l}
h\left(\bar{x}, \mathbf{k}_{u}\right) \leqslant 0 \\
A_{i n} \mathbf{k}_{u} \leq b_{i n}+B_{i n} \bar{x}
\end{array}\right.
$$

- Dual feasibility:

$$
H \mathbf{k}_{u}+F^{T} \bar{x}+A_{i n}^{T} \mu+\nabla_{\mathbf{k}_{u}} h\left(\bar{x}, \mathbf{k}_{u}\right)^{T} \nu=0 ; \mu \geqslant 0, \nu \geqslant 0
$$

- Complementary slackness:

$$
\left[\mu^{T} \nu^{T}\right]\left[\begin{array}{c}
A_{i n} \mathbf{k}_{u}-B_{i n} \bar{x}-b_{i n} \\
h\left(\bar{x}, \mathbf{k}_{u}\right)
\end{array}\right]=0
$$

The difference resides in the fact that the KKT conditions are only necessary and not sufficient for optimality due to the presence of nonlinearity.

\subsection{The topology of the feasible domain}

Indeed the sufficiency is lost due to the lack of constraint qualification (the Abadie constraint qualification holds automatically for the linear constraints but needs additional assumptions for the nonlinear case, see the next theorem).

Theorem (KKT sufficient conditions) [10]: Let $x=\bar{x}$ and the associated feasible domain $\mathbf{U}(\bar{x})$ be a nonempty set in $\mathbb{R}^{N m}$ described by the constraints in $(15)$, with $h_{i}\left(\mathbf{k}_{u}\right)=h_{i}\left(\bar{x}, \mathbf{k}_{u}\right): \mathbb{R}^{N m} \rightarrow \mathbb{R}$, the components of $h\left(\mathbf{k}_{u}\right)$. Let $\mathbf{k}_{u}^{*} \in$ 
$\mathbf{U}(\bar{x})$ and let $\left.\mathcal{I}=\left\{i: h_{i}\left(\bar{x}, \mathbf{k}_{u}^{*}\right)=0\right\}, \mathcal{J}=\left\{j: A_{i n_{j}} \mathbf{k}_{u}^{*}-B_{i n_{j}} \bar{x}-b_{i n_{j}}=0\right)\right\}$. Suppose the KKT conditions hold, such that:

$$
H \mathbf{k}_{u}^{*}+F^{T} \bar{x}+\sum \mu_{j} A_{i n_{j}}^{T}+\sum \gamma_{i} \nabla_{\mathbf{k}_{u}} h_{i}\left(\bar{x}, \mathbf{k}_{u}^{*}\right)^{T}=0
$$

If $h_{i}$ is quasiconvex at $\mathbf{k}_{u}^{*} \forall i \in \mathcal{I}$, then this represents a global solution to (15) $\square$

Due to these problems, up to date, the explicit solutions for the general nonlinear multiparametric programming case were not tackled. Only for convex nonlinearities approximate explicit solutions were proposed [16]. In the following a solution based on linear approximation of feasible domains is proposed. This will answer the question regarding the optimality of a solution with piecewise linear structure.

\subsection{Preliminaries for linear approximations of mixed linear/nonlinear feasible domains}

The idea is to exploit the existence of linear constraints in (15) and construct exact solutions as long as the unconstrained optimum can be projected on them. In a second stage if the unconstrained optimum is projected on the convex part of the nonlinear constraints, then an approximate solution is obtained by their linearization. Finally if the unconstrained optimum has to be projected on the nonconvex constraints then a Voronoi partition is used to construct the explicit solution. Before detailing the algorithms several useful tools are introduced.

Gridding of the parameter space: The parameters (state) space is sampled in order to obtain a representative grid $\mathcal{G}$. The way of distributing the points in the state space may follow a uniform distribution, logarithmic or tailored according to the a-priori knowledge of the nonlinearities.

For each point of the grid $x \in \mathcal{G}$ a set of points on the frontier of the feasible domain $D(x)$ can be obtained $-\mathcal{V}_{x}$ by the same kind of parceling. By collecting $\mathcal{V}_{x}$ for all $x \in \mathcal{G}$ a distribution of points $\mathcal{V}_{\mathcal{G}}$ in the extended arguments+parameters space is obtained.

Convex hulls: A basic operation is the construction of the convex hull (or a adequate approximation) for the feasible domain in (15). Writing this parameterized feasible domain as:

$$
D(x)=\left\{\mathbf{k}_{u} \mid h\left(x, \mathbf{k}_{u}\right) \leqslant 0 ; A_{i n} \mathbf{k}_{u} \leq b_{i n}+B_{\text {in }} x\right\}
$$

and using the distribution of points on the frontier $\mathcal{V}_{\mathcal{G}}$, one can define in the extended (argument+parameters) space a convex hull $\mathcal{C}_{\mathcal{V}_{\mathcal{G}}}$ :

$$
\begin{gathered}
\mathcal{C}_{\mathcal{V}_{\mathcal{G}}}=\left\{\left[\begin{array}{c}
\mathbf{k}_{u} \\
x
\end{array}\right] \in \mathbb{R}^{m N+n} \mid \exists\left[\begin{array}{c}
\mathbf{k}_{u_{i}} \\
x_{i}
\end{array}\right], i=1 . . m N+n+1, \mathbf{k}_{u_{i}} \in \mathcal{V}_{\mathcal{G}},\right. \\
\text { s.t. } \left.\left[\begin{array}{c}
\mathbf{k}_{u} \\
x
\end{array}\right]=\sum_{i=1}^{m+n+1} \lambda_{i}\left[\begin{array}{c}
\mathbf{k}_{u_{i}} \\
x_{i}
\end{array}\right], \sum_{i=1}^{m N+n+1} \lambda_{i}=1 ; \lambda_{i} \geq 0\right\}
\end{gathered}
$$

Voronoi partition: The Voronoi partition is the decomposition of a metric space $\mathbb{R}^{n}$ in regions associated with a specified discrete set of points. 
Let $S=\left\{s_{1}, s_{2}, \ldots, s_{\nu}\right\}$ be a collection of $\nu$ points in $\mathbf{R}^{n}$. For each point $s_{i}$ a set $V_{i}$ is associated such that $\bigcup_{i} V_{i}=\mathbf{R}^{n}$. The definition of $V_{i}$ will be:

$$
V_{i}=\left\{x \in \mathbf{R}^{n} \mid\left\|x-v_{i}\right\|_{2} \leq\left\|x-v_{i}\right\|_{2}, \forall j \neq i\right\}
$$

It can be observed that each frontier of $V_{i}$ is part of the bisection hyperplane between $s_{i}$ and one of the neighbor points $s_{j}$. As a consequence of this fact, the regions $V_{i}$ are polyhedrons. Globally, the Voronoi partition is a decomposition of space $\mathbf{R}^{n}$ in $\nu$ polyhedral regions.

\subsection{Nonparameterized case}

In the following $\mathfrak{F}(X)$ (and $\mathfrak{I n t}(X)$ ) represents the frontier (and the interior respectively) for a compact set $X$.

Consider the nonparameterized optimization problem:

$$
\begin{gathered}
\mathbf{k}_{u}^{*}=\underset{\mathbf{k}_{u}}{\arg \min } 0.5 \mathbf{k}_{u}^{T} \mathbf{k}_{u}+c^{T} \mathbf{k}_{u} \\
\left\{\begin{array}{c}
h\left(\mathbf{k}_{u}\right) \leqslant 0 \\
A_{\text {in }} \mathbf{k}_{u} \leq b_{\text {in }}+B_{\text {in }} x
\end{array}\right.
\end{gathered}
$$

In relation with the feasible domain $D$ of 23 we define:

$\mathfrak{R}_{L}(D)$ The set of linear constraints in the definition of $D$

$\mathfrak{R}_{N L}(D)$ The set of nonlinear constraints in the definition of $D$

$\mathfrak{S}\left(\mathfrak{R}_{*}, \mathbf{k}_{u}\right)$ The subset of constraints in $\mathfrak{R}_{*}$ (either $\mathfrak{R}_{L}$, either $\mathfrak{R}_{N L}$ ) saturated by the vector $\mathbf{k}_{u}$

$\mathfrak{B}\left(\mathfrak{R}_{*}, \mathbf{k}_{u}\right)$ The subset of constraints in $\mathfrak{R}_{*}$ violated by the vector $\mathbf{k}_{u}$

Algorithm:

1. Obtain a set of points $(\mathcal{V})$ on the frontier of the feasible domain $D$

2. Construct the convex hull $\mathcal{C}_{\mathcal{V}}$

3. Split the set $\mathcal{V}$ as $\widetilde{\mathcal{V}} \cup \overline{\mathcal{V}}_{L} \cup \overline{\mathcal{V}}_{N L} \cup \widehat{\mathcal{V}}$

$-\widetilde{\mathcal{V}} \in \mathfrak{F}\left(\mathcal{C}_{\mathcal{V}}\right)$ and $\mathcal{C}_{\mathcal{V}}=\mathcal{C}_{\mathcal{V} \backslash \tilde{\mathcal{V}}}$ (in words, $\widetilde{\mathcal{V}}$ contains those points in $\mathcal{V}$ which lie on the frontier of $\mathcal{C}_{\mathcal{V}}$ but are not vertices);

$-\mathcal{V}_{L} \in \mathcal{V} \backslash \widetilde{\mathcal{V}}, \mathcal{V}_{L} \in \mathfrak{F}\left(\mathcal{C}_{\mathcal{V}}\right)$ and $\mathcal{V}_{L}$ saturates at least one linear constraint

- $\mathcal{V}_{N L} \in \mathcal{V} \backslash \widetilde{\mathcal{V}}, \mathcal{V}_{N L} \in \mathfrak{F}\left(\mathcal{C}_{\mathcal{V}}\right)$ and $\mathcal{V}_{N L}$ saturates only nonlinear constraints

$-\widehat{\mathcal{V}} \in \mathfrak{I n t}\left(\mathcal{C}_{\mathcal{V}}\right)$

4. Construct the dual representation of $\mathcal{C}_{\mathcal{V}}$. This will be represented as an intersection of halfspaces $\mathcal{H}$.

5. Split $\mathcal{H}$ in $\overline{\mathcal{H}} \cup \widehat{\mathcal{H}}$

$-\widehat{\mathcal{H}} \subset \mathcal{H}$ such that $\exists x \in \mathcal{C}_{\mathcal{V}}$ with $\mathfrak{S}(\widehat{\mathcal{H}}, x) \neq \emptyset$ and $\mathfrak{B}\left(\Re_{N L}, x\right) \neq \emptyset$

$-\overline{\mathcal{H}}=\mathcal{H} \backslash \widehat{\mathcal{H}}$

6. Project the unconstrained optimum $\mathbf{k}_{u}=-c$ on $\mathcal{C}_{\mathcal{V}}$ :

$$
\mathbf{k}_{u}^{*} \leftarrow \operatorname{Proj}_{\mathcal{C}_{\mathcal{V}}}\{-c\}
$$


7. If $\mathbf{k}_{u}^{*}$ saturates a subset of constraints $\mathcal{K} \subset \widehat{\mathcal{H}}$

(a) Retain the set of points:

$$
S=\left\{v \in \widehat{\mathcal{V}} \mid \forall \mathbf{k}_{u} \in \mathcal{C}_{\mathcal{V}} \text { s.t. } \mathfrak{S a t}\left(\widehat{\mathcal{H}}, \mathbf{k}_{u}\right)=\mathcal{K} ; \quad \mathfrak{B}\left(\mathfrak{R}_{N L}, \mathbf{k}_{u}\right)=\mathfrak{S a t}\left(\mathfrak{R}_{N L}, v\right)\right\}
$$

(b) Construct the Voronoi partition for the collection of points in $S$

(c) Position $\mathbf{k}_{u}^{*}$ w.r.t. this partition and map the suboptimal solution $\mathbf{k}_{u}^{*} \leftarrow v$ where $v$ is the vertex corresponding to the active region

8. If the quality of the solution is not satisfactory, improve the distribution of the points $\mathcal{V}$ by augmenting the resolution around $\mathbf{k}_{u}^{*}$ and restart from (2).

\subsection{Explicit solution - taking into account the parametrization}

Consider now the multiparametric optimization:

$$
\mathbf{k}_{u}^{*}=\underset{\mathbf{k}_{u}}{\arg \min } 0.5 \mathbf{k}_{u}^{T} H \mathbf{k}_{u}+\mathbf{k}_{u}^{T} F x
$$

and the feasible combinations defined by the set:

$$
D=\left\{\left[\begin{array}{c}
\mathbf{k}_{u} \\
x
\end{array}\right] \in \mathbb{R}^{m N+n} \mid \begin{array}{c}
h\left(x, \mathbf{k}_{u}\right) \leqslant 0 \\
A_{i n} \mathbf{k}_{u} \leq b_{i n}+B_{i n} x
\end{array}\right\}
$$

Algorithm:

1. Grid the parameters space $\mathbb{R}^{n}$ and retain the feasible nodes $\mathcal{G}$

2. Obtain in the extended argument+parameters space a set of points $\left(\mathcal{V}_{\mathcal{G}}\right)$ lying on the frontier of $D$

3. Construct the convex hull $\mathcal{C}_{\mathcal{V}}$ for the points in $\mathcal{V}_{\mathcal{G}}$

4. Split the set $\mathcal{V}_{\mathcal{G}}$ as $\widetilde{\mathcal{V}} \cup \overline{\mathcal{V}}_{L} \cup \overline{\mathcal{V}}_{N L} \cup \widehat{\mathcal{V}}$

$-\widetilde{\mathcal{V}} \in \mathfrak{F}\left(\mathcal{C}_{\mathcal{V}}\right)$ and $\mathcal{C}_{\mathcal{V}}=\mathcal{C}_{\mathcal{V} \backslash \widetilde{\mathcal{V}}}$ (in words, $\widetilde{\mathcal{V}}$ contains those points in $\mathcal{V}$ which lie on the frontier of $\mathcal{C}_{\mathcal{V}}$ but are not vertices);

$-\mathcal{V}_{L} \in \mathcal{V}_{\mathcal{G}} \backslash \tilde{\mathcal{V}}, \mathcal{V}_{L} \in \mathfrak{F}\left(\mathcal{C}_{\mathcal{V}}\right)$ and $\mathcal{V}_{L}$ saturates at least one linear constraint

$-\mathcal{V}_{N L} \in \mathcal{V}_{\mathcal{G}} \backslash \tilde{\mathcal{V}}, \mathcal{V}_{N L} \in \mathfrak{F}\left(\mathcal{C}_{\mathcal{V}}\right)$ and $\mathcal{V}_{N L}$ saturates only nonlinear constraints

$-\widehat{\mathcal{V}} \in \mathfrak{I n t}\left(\mathcal{C}_{\mathcal{V}}\right)$

5. Construct the dual representation of $\mathcal{C}_{\mathcal{V}}$. This will be represented as a intersection of halfspaces $\mathcal{H}$.

6. Split $\mathcal{H}$ in $\overline{\mathcal{H}} \cup \widehat{\mathcal{H}}$

- $\widehat{\mathcal{H}} \subset \mathcal{H}$ such that $\exists x \in \mathcal{C}_{\mathcal{V}}$ with $\mathfrak{S}(\widehat{\mathcal{H}}, x) \neq \emptyset$ and $\mathfrak{B}\left(\mathfrak{R}_{N L}, x\right) \neq \emptyset$

$-\overline{\mathcal{H}}=\mathcal{H} \backslash \widehat{\mathcal{H}}$

7. Project the set

$$
U=\left\{\left[\begin{array}{c}
\mathbf{k}_{u} \\
x
\end{array}\right] \mid\left[\begin{array}{c}
\mathbf{k}_{u} \\
x
\end{array}\right]=\left[\begin{array}{c}
H^{-1} F \\
I
\end{array}\right] x, \forall x \in \mathbb{R}^{n}\right\}
$$

on $\mathcal{C}_{\mathcal{V}}$ :

$$
U^{*} \leftarrow \operatorname{Proj}_{\mathcal{C}_{\mathcal{V}}} U
$$


8. If $\exists x_{0}$ such that the point:

$$
\left[\begin{array}{c}
\mathbf{k}_{u}^{*} \\
x_{0}
\end{array}\right]=U^{*} \cap\left\{\left[\begin{array}{c}
\mathbf{k}_{u} \\
x
\end{array}\right] \mid x=x_{0}\right\}
$$

saturates a subset of constraints

$$
\mathcal{K}\left(x_{0}\right)=\mathfrak{S}\left(\mathcal{H},\left[\begin{array}{l}
\mathbf{k}_{u} \\
x_{0}
\end{array}\right]\right) \subset \widehat{\mathcal{H}}
$$

then:

(a) Construct

$$
U_{N L}\left(x_{0}\right)=\left\{\left[\begin{array}{c}
\mathbf{k}_{u} \\
x
\end{array}\right] \in U \mid\left[\begin{array}{c}
\mathbf{k}_{u}^{*} \\
x
\end{array}\right] \in U^{*} \text { s.t. } \quad \mathfrak{S}\left(\mathcal{H},\left[\begin{array}{l}
\mathbf{k}_{u} \\
x_{0}
\end{array}\right]\right)=\mathcal{K}\left(x_{0}\right)\right\}
$$

(b) Perform:

$$
U^{*}=U^{*} \backslash\left\{\left[\begin{array}{c}
\mathbf{k}_{u} \\
x
\end{array}\right] \mid \mathfrak{S}\left(\mathcal{H},\left[\begin{array}{c}
\mathbf{k}_{u} \\
x_{0}
\end{array}\right]\right)=\mathcal{K}\left(x_{0}\right)\right\}
$$

(c) Retain the set of points:

$$
S=\left\{v \in \widehat{\mathcal{V}} \mid \forall\left[\begin{array}{c}
\mathbf{k}_{u} \\
x
\end{array}\right] \in \mathcal{C}_{\mathcal{V}} \quad \begin{array}{l}
\text { with } \mathfrak{S}\left(\widehat{\mathcal{H}},\left[\begin{array}{c}
\mathbf{k}_{u} \\
x
\end{array}\right]\right)=\mathcal{K}\left(x_{0}\right) \Rightarrow \\
\left.\mathfrak{B}\left(\mathfrak{R}_{N L}, x\right) \stackrel{S}{=}\left(\Re_{N L}, v\right)\right\}
\end{array}\right.
$$

(d) Construct the Voronoi partition for the collection of points in $S$

(e) Position $U_{N L}\left(x_{0}\right)$ w.r.t. this partition and map the suboptimal solution $U_{N L}^{*}\left(x_{0}\right) \leftarrow U_{N L}\left(x_{0}\right)$ by using the vertex $v$ for each active region.

$$
\left[\begin{array}{c}
\mathbf{k}_{u}^{*} \\
x
\end{array}\right]=v \leftarrow\left[\begin{array}{c}
\mathbf{k}_{u} \\
x
\end{array}\right]
$$

else: jump to (10)

9. Return to point (8)

10. If the quality of the solution is not satisfactory, improve the distribution of the points $\mathcal{V}_{\mathcal{G}}$ and restart from (2).

\section{Numerical example}

Consider the MPC problem implemented using the first control action of the optimal sequence:

$$
k_{u}^{*}=\underset{k_{u}}{\arg \min } \sum_{i=0}^{N-1} x_{t+k \mid t}^{T} Q x_{t+k \mid t}+u_{t+k \mid t}^{T} R u_{t+k \mid t}+x_{t+N \mid t}^{T} P x_{t+N \mid t}
$$




$$
\left\{\begin{array}{l}
x_{t+k+1 \mid t}=\left[\begin{array}{ll}
1 & 1 \\
0 & 1
\end{array}\right] x_{t+k \mid t}+\left[\begin{array}{ll}
1 & 0 \\
2 & 1
\end{array}\right] u_{t+k \mid t} k \geqslant 0 \\
{\left[\begin{array}{l}
-2 \\
-2
\end{array}\right] \leqslant u_{t+k \mid t} \leqslant\left[\begin{array}{l}
2 \\
2
\end{array}\right], \sqrt{\left(u_{t+k \mid t}^{1}\right)^{2}+\left(u_{t+k \mid t}^{2} \pm 2\right)^{2}} \geqslant \sqrt{3} ; \quad 0 \leqslant k \leqslant N_{u}-1} \\
u_{t+k \mid t}=\underbrace{\left[\begin{array}{cc}
0.59 & 0.76 \\
-0.42 & -0.16
\end{array}\right]}_{K_{L Q R}} x_{t+k \mid t} N_{u} \leqslant k \leqslant N_{y}-1
\end{array}\right.
$$

with

$$
Q=\left[\begin{array}{rr}
10 & 0 \\
0 & 1
\end{array}\right] ; R=\left[\begin{array}{ll}
2 & 0 \\
0 & 3
\end{array}\right] ; P=\left[\begin{array}{rr}
13.73 & 2.46 \\
2.46 & 2.99
\end{array}\right] ; N_{u}=1 ; N=2
$$

By following the previous algorithm, in the first stage, the partition of the state space is performed by considering only the linear constraints (figure 1(a)). Each such region corresponds with a specific projection law. By simply verifying the regions where this projection law obeys the nonlinear constraints, the exact part of the explicit solution is obtained (figure 1(b)).

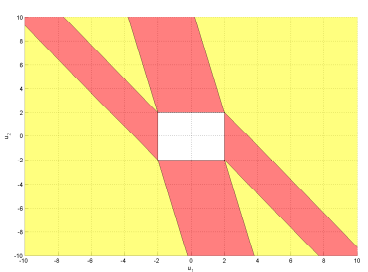

(a)

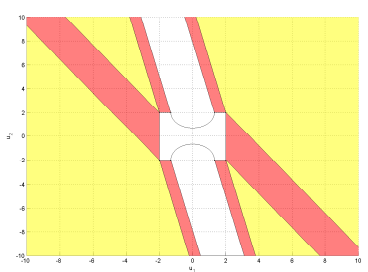

(b)

Fig. 1. a) Partition of the arguments space (linear constraints only). b) Retention of the regions with feasible linear projections.

Further, a distribution of points on the nonlinear frontier of the feasible domain has to be obtained with the associated Voronoi partition. By superposing it to the regions non covered at the previous step a complete partition of the arguments space is realized. Figures 2(a)-2(a) depict such a partitions for 10 and 100 points for each nonlinear constraint.

By correspondence, the figures 3(a) and 3(b) describe the partition of the state space for the explicit solution. Finally the complete explicit solution for the two cases are described in figures 4(a) and 4(b). The discontinuities are observable as well as the increase in resolution over the nonlineairity with the augmentation of the number of points in the Voronoi partition. In order to give an image of the complexity it must be said that the explicit solutions have 31 and 211 regions respectively and the computational effort was less than $2 s$ in the first case and $80 \mathrm{~s}$ in the second case, mainly spent in the construction of supplementary regions in the Voronoi partition. 


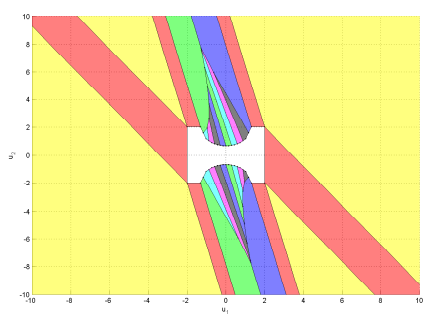

(a)

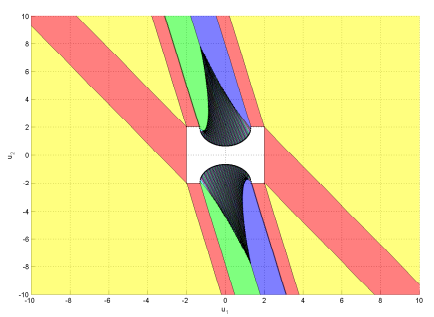

(b)

Fig. 2. Partition of the arguments space (nonlinear case) - a) 10 points per active nonlinear constraint; b) 100 points per nonlinear constraint.

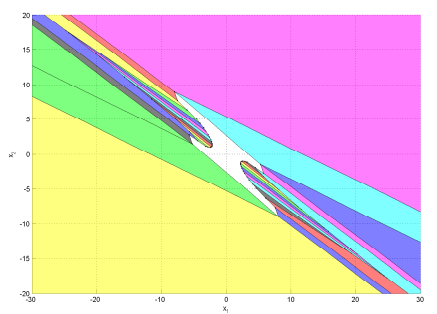

(a)

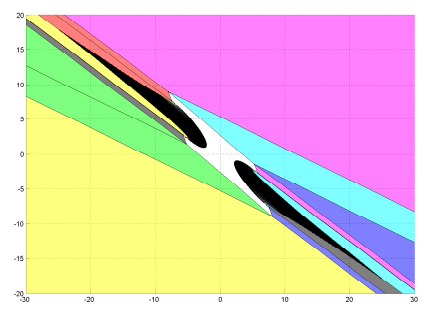

(b)

Fig. 3. Partition of the state space - a) 10 points per nonlinear constraint; b) 100 points per nonlinear constraint.

\section{Conclusion}

The parameterized polyhedra offer a transparent characterization of the MPC degrees of freedom. Once the complete description of the feasible domain as a parameterized polyhedron is obtained, explicit MPC laws can be constructed using the projection of the unconstrained optimum. The topology of the feasible domain can lead to explicit solution even if nonlinear constraints are taken into consideration. The price to be paid is found in the degree of suboptimality.

\section{References}

1. Scokaert, P.O., Mayne, D.Q., Rawlings, J.B.: Suboptimal model predictive control (feasibility implies stability). In: IEEE Transactions on Automatic Control. Volume 44. (1999) 648-654

2. Olaru, S., Dumur, D.: A parameterized polyhedra approach for explicit constrained predictive control. In: 43rd IEEE Conference on Decision and Control, 2004. 1580$1585 \mathrm{Vol} .2$ 


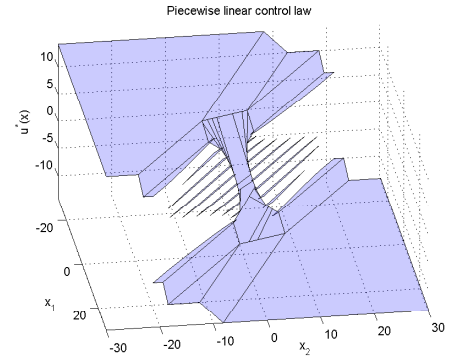

(a)

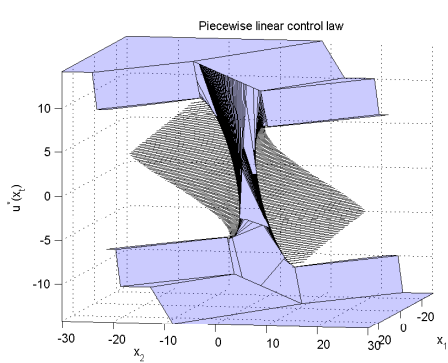

(b)

Fig. 4. Explicit control law - a) 10 points per nonlinear constraint; b) 100 points per nonlinear constraint.

3. Motzkin, T.S., R.H.T.G., R.M., T.: The Double Description Method, republished in Theodore S. Motzkin: Selected Papers, (1983). Birkhauser (1953)

4. Schrijver, A.: Theory of Linear and Integer Programming. John Wiley and Sons, NY (1986)

5. Leverge, H.: A note on chernikova's algorithm. In: Technical Report 635, IRISA, France (1994)

6. Loechner, V., Wilde, D.K.: Parameterized polyhedra and their vertices. International Journal of Parallel Programming V25(6) (December 1997) 525-549

7. Olaru, S., Dumur, D.: Compact explicit mpc with guarantee of feasibility for tracking. In: 44th IEEE Conference on Decision and Control, and European Control Conference. (2005) 969-974

8. Seron, M., Goodwin, G., Dona, J.D.: Characterisation of receding horizon control for constrained linear systems. In: Asian Journal of Control. Volume 5. (2003) 271-286

9. Bemporad, A., Morari, M., Dua, V., Pistikopoulos, E.: The Explicit Linear Quadratic Regulator for Constrained Systems. Automatica 38(1) (2002) 3-20

10. Goodwin, G., Seron, M., Dona, J.D.: Constrained Control and Estimation. Springer, Berlin (2004)

11. Borelli, F.: Constrained Optimal Control of Linear and Hybrid Systems. SpringerVerlag, Berlin (2003)

12. Tondel, P., Johansen, T., Bemporad, A.: Evaluation of piecewise affine control via binary search tree. Automatica 39 (2003) 945-950

13. Bemporad, A., Borrelli, F., Morari, M.: Robust Model Predictive Control: Piecewise Linear Explicit Solution. In: European Control Conference. (2001) 939-944

14. Kerrigan, E., Maciejowski, J.: Feedback min-max model predictive control using a single linear program: Robust stability and the explicit solution. International Journal of Robust and Nonlinear Control 14(4) (2004) 395-413

15. Olaru, S., Dumur, D.: On the continuity and complexity of control laws based on multiparametric linear programs. In: 45th IEEE Conference on Decision and Control. (2006)

16. Grancharova, A., Tondel, P., Johansen, T.A.: International Workshop on Assessment and Future Directions of Nonlinear Model Predictive Control. (2005) 